STUDIES ON COLLATERAL CIRCULATION. II. THERMIC CHANGES AFTER ARTERIAL LIGATION, SECTION OF SPINAL CORD OR POSTERIOR ROOTS AND GANGLIONECTOMY

\author{
By SAMUEL C. HARVEY AND BÉLA HALPERT \\ (From the Department of Surgery, Yale University School of Medicine, New Haven)
}

(Received for publication February 26, 1931)

Previous experiments (1) have shown that in the dog ligation of both external iliac arteries close to their origin caused a decrease in the temperature of both hind feet to room temperature and that cutting of the rami communicantes and removal of the tributary sympathetic ganglia caused a rise in temperature of the corresponding foot to about that before ligation. The present experiments were planned to ascertain $(a)$ whether division of the spinal cord at about the level of the first lumbar vertebra, and $(b)$ section of the posterior roots below this level would influence the above reaction.

\title{
METHOD
}

As in the previous experiments, the animal was given sodium amytal [Lilly] intraperitoneally and attached to the Leeds and Northrup temperature recorder by thermopiles about half an hour later. After an equilibrium had been established, both external iliac arteries were exposed and ligated as in the previous experiments. In experiments $(a)$ after the temperature of both feet had decreased to about room temperature and had remained there for several hours, the spinal cord was sectioned after laminectomy at about the level of the first lumbar vertebra. After sufficient time had elapsed for a reaction to appear, were it going to do so, the rami communicantes of the tributary sympathetic ganglia of one limb were severed and the ganglia were removed. In experiments $(b)$ when the temperature of both feet had decreased to about room temperature after ligation of both external iliac arteries and had remained there for several hours, 
all posterior roots below the level of the first lumbar vertebra were cut intradurally on one side after laminectomy. After sufficient time had elapsed for a reaction to appear, were it going to do so, the rami communicantes of the tributary sympathetic ganglia of the corresponding limb were removed.

\section{EXPERIMENTAL DATA}

Ligation of both external iliac arteries close to their origin from the aorta was followed by decrease in temperature of both feet to room temperature. Transection of the spinal cord at the level of the first lumbar vertebra exerted no influence whatsoever upon the lowered. temperature of the feet. In every case unilateral transection of the rami communicantes and removal of the tributary sympathetic ganglia were promptly followed by increase in temperature of the corresponding foot from room temperature to about that of the foot before ligation.

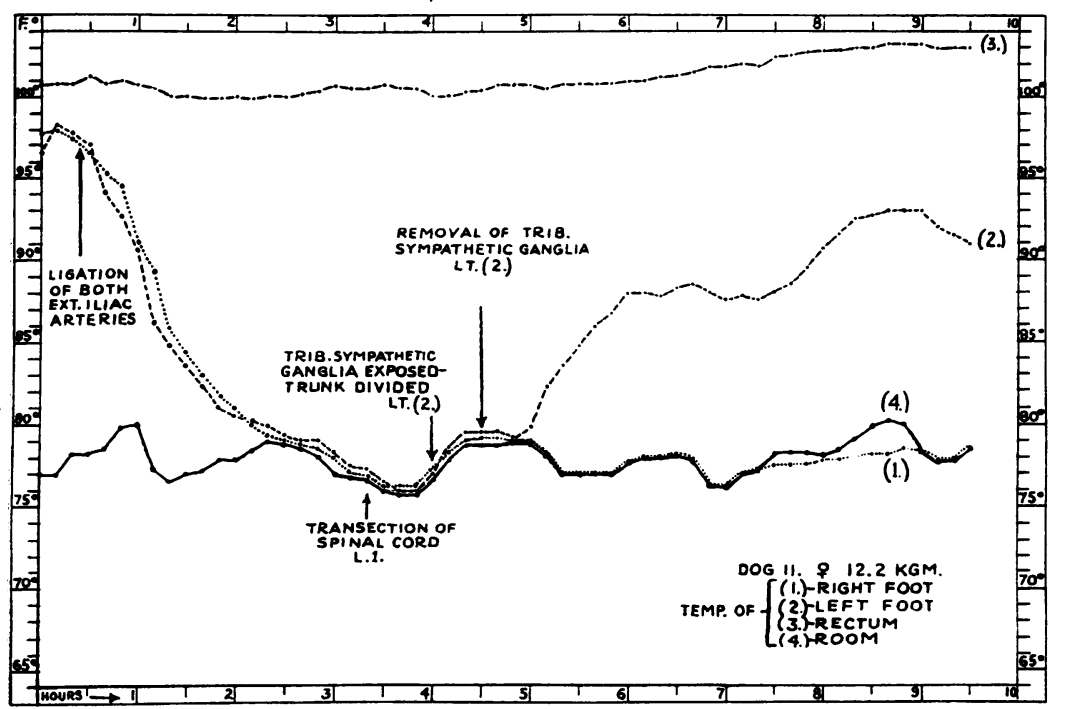

Fig. 1. Following Ligation of Both External Iliac Arteries the Temperature of Both Feet Dropped $20^{\circ} \mathrm{F}$. Transection of the spinal cord at the level of the first lumbar vertebra had no influence whatsoever upon this lowered temperature. Unilateral transection of the rami communicantes and removal of the tributary sympathetic ganglia caused a prompt rise in temperature of the corresponding foot of $15^{\circ} \mathrm{F}$., i.e., from room temperature to nearly that of the foot before ligation. 
Transection of all posterior roots below the level of the first lumbar vertebra on one side exerted no influence whatsoever upon the temperature of the foot which had dropped to room temperature after ligation of its external iliac artery. Subsequent transection of the rami communicantes and removal of the tributary sympathetic ganglia were promptly followed by increase in temperature of the corresponding foot from room temperature to nearly that of the foot before ligation.

Figures 1 and 2 illustrate the phenomena described.

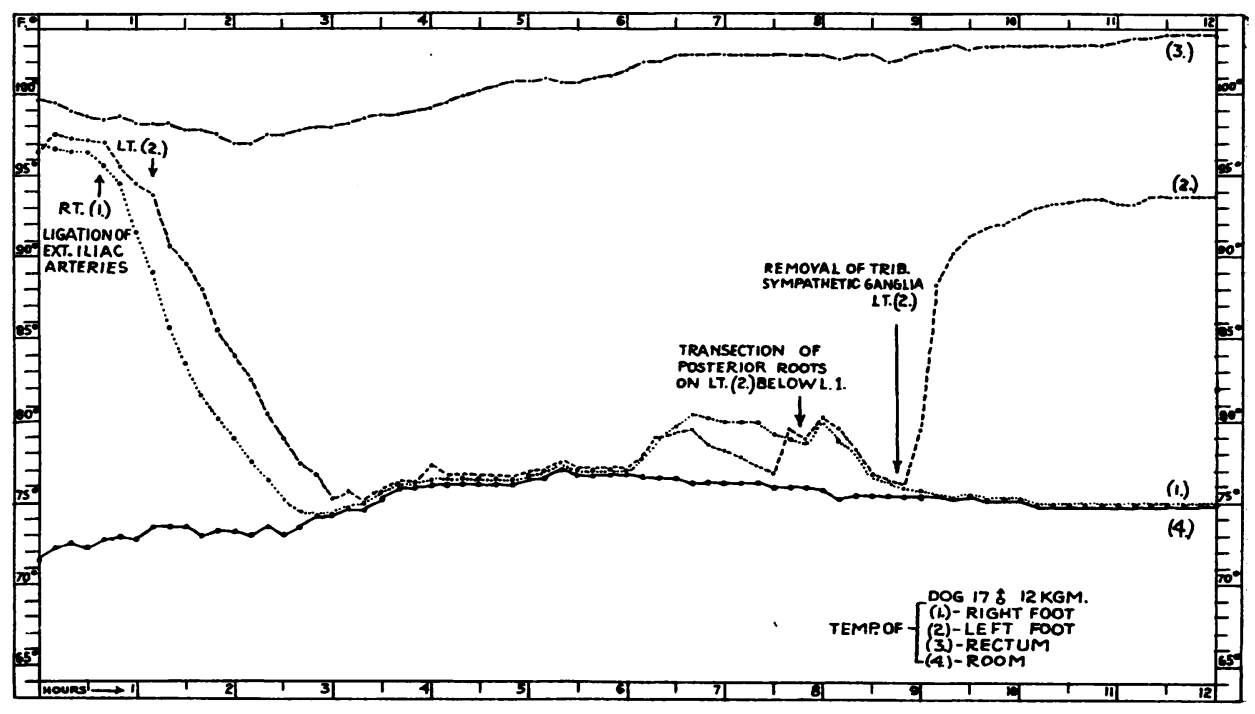

Fig. 2. Following Ligation of Both External Iliac Arteries the Temperature of Both Feet Dropped $20^{\circ} \mathrm{F}$. Transection of all posterior roots on one side below the level of the first lumbar vertebra had no influence whatsoever upon the lowered temperature of the foot. Subsequent transection of the rami communicantes and removal of the tributary sympathetic ganglia caused a prompt rise in temperature of the corresponding foot of $19^{\circ}$ F., i.e., from room temperature to nearly that of the foot before ligation.

\section{COMMENT}

Under the above experimental conditions transection of the spinal cord at the level of the first lumbar vertebra has no effect whatsoever upon the lowered temperature of the feet produced by ligation of both external iliac arteries. Subsequent removal of the tributary sympathetic ganglia, on the other hand, is followed by a prompt rise in 
temperature of the corresponding limb from room temperature to nearly that of the foot before ligation. This occurred in exactly the same way as in the previous experiments in which the cord was not transected. These observations suggest that in these experiments bulbar impulses play no appreciable part in the mechanism of the development of collateral circulation.-Transection of all posterior roots below the level of the first lumbar vertebra on one side had no effect whatsoever upon the lowered temperature of the feet produced by ligation of both external iliac arteries. Removal of the tributary sympathetic ganglia of the corresponding limb was followed by a prompt rise in temperature of that limb in much the same manner as in those experiments in which the posterior roots had not been transected. These observations suggest that impulses communicating through the posterior roots below the level of the first lumbar vertebra play no appreciable part in the mechanism of the reestablishment of former conditions in the limb by collateral circulation, as observed in these experiments.

\section{SUMMARY AND CONCLUSIONS}

In the dog the lowered temperature of the feet produced by ligation of both external iliac arteries was not influenced by either (a) transection of the spinal cord at the level of the first lumbar vertebra or $(b)$ transection of all the posterior roots on one side below the level of the first lumbar vertebra. Removal of the tributary sympathetic ganglia caused a prompt rise in temperature of the corresponding foot from room temperature to nearly that of the limb before ligation whether or not the spinal cord had been transected at the level of the first lumbar vertebra, or whether or not all posterior roots below this level on the corresponding side had been transected.

These observations suggest that: (a) bulbar impulses and (b) impulses communicating through the posterior roots below the level of the first lumbar vertebra play no appreciable part in the mechanism of the development of collateral circulation, as observed in these experiments.

\section{BIBLIOGRAPHY}

1. Mulvihill, Daniel A., and Harvey, Samuel C., J. Clin. Invest., 1931, $\mathrm{x}, 423$. Studies on Collateral Circulation. I. Thermic Changes after Arterial Ligation and Ganglionectomy. 